\title{
Delayed Diagnosis of Cauda Eqina Syndrome with Perineural Cyst after Combined Spinal-Epidural Anesthesia in Hemodialysis Patient
}

\author{
Shigeo Ishiguro ${ }^{1}$, Koji Akeda ${ }^{2}$, Masaya Tsujii ${ }^{2}$, Akihiro Sudo ${ }^{2}$ \\ ${ }^{1}$ Department of Orthopaedic Surgery, Oyamada Memorial Spa Hospital, Yokkaichi, Japan \\ ${ }^{2}$ Department of Orthopaedic Surgery, Mie Universtiy Graduate School of Medicine, Tsu, Japan
}

\begin{abstract}
Symptomatic Tarlov (perineural cysts) are uncommon. In the following hemodialysis case, cauda equina syndrome was not detected after combined spinal-epidural anesthesia untilthe patient reported a lack of sensation in the perianal area 14 days postoperatively. She had normal motor function of her extremities. A laminectomy and cyst irrigation was performed. After the operation, her sphincter disturbance subsided gradually and her symptoms had disappeared.
\end{abstract}

Keywords: Cauda eqina syndrome; Perineural cyst; Hemodialysis

\section{Introduction}

Tarlov cysts or perineural cysts have become a common incidental finding during lumbosacral magnetic resonance (MR) imaging $[1,2]$. They are usually harmless lesions of the nerve roots located at the sacral level, of uncertain etiology. Most Tarlov cysts remain asymptomatic with no clinical relevance and the usual symptoms are pain or radiculopathy $[1,3]$. Some studies have reported cases of urinary incontinence $[4,5]$. This case was a dialysis patient who produced no urine, therefore cauda equina syndrome could not be detected by urinary incontinence. This is a case report that extremely rare postanesthetic complication in the hemodialysis female was diagnosed at the last moment and treated successfully.

\section{Case Report}

A 53-year-old female who had been on maintenance hemodialysis due to diabetes mellitus developed an infection in her left femoral prosthesis and needed removal surgery.

In consideration of her general status, general anesthesia was not undertaken. We performed removal surgery successfully under combined spinal-epidural anesthesia by puncturing L3/4, $3 \mathrm{~mL}$ of $0.5 \%$-bupivacaine was administered into the subarachoid cavity and continuous injection of 2\%-mepivacaine was performed at a rate of $3.3 \mathrm{~mL}$ per hour for 24 hours postoperatively. Pain relief was quite satisfactory during and after operation as this combination technique provides a rapid onset and spinal block reliability with high-quality analgesia by supplemented by the epidural catheter during and after surgery. Postoperative rehabilitation was started on the seventh

Received Mar 5, 2012; Revised May 2, 2012; Accepted May 9, 2012

Corresponding author: Shigeo Ishiguro

Department of Orthopaedic Surgery, Oyamada Memorial Spa Hospital,

5538-1 Yamada-cho, Yokkaichi Mie 512-1111, Japan

Tel: +81-59-328-1260, Fax: +81-59-328-3040, E-mail: stoneblack1496@syutaikai.jp 
postoperative day as no paresis of the four extremities was observed.

For the first time, on the 14th postoperative day, she complained that she was feeling hypoesthesia in the perianal area and loss of stool sensation. The client noted that she recognized the inability to sense stool but hesitated to inform us due to embarrassment. She seemed to manifest the symptom of fecal incontinence.

We immediately conducted neurological and radiological examinations to determine the source of her symptoms.
The plain MRI revealed at least two perineural cysts inside the spinal canal at the L5/S1 level, that were considered to be S2 root cysts (Fig. 1). The myelography showed a blockade of the contrast medium (Fig. 2). Afterward, the computed tomography was taken which showed a delayed filling of the right S2 root cysts and an inflated dural sheath of the S2 or S3 roots which could be expressed as cysts.

We supposed that her symptoms were caused by a volume effect or a prolonged analgesic effect entrapped in root cysts $[3,6]$. We conducted an S1 to S5 laminectomy

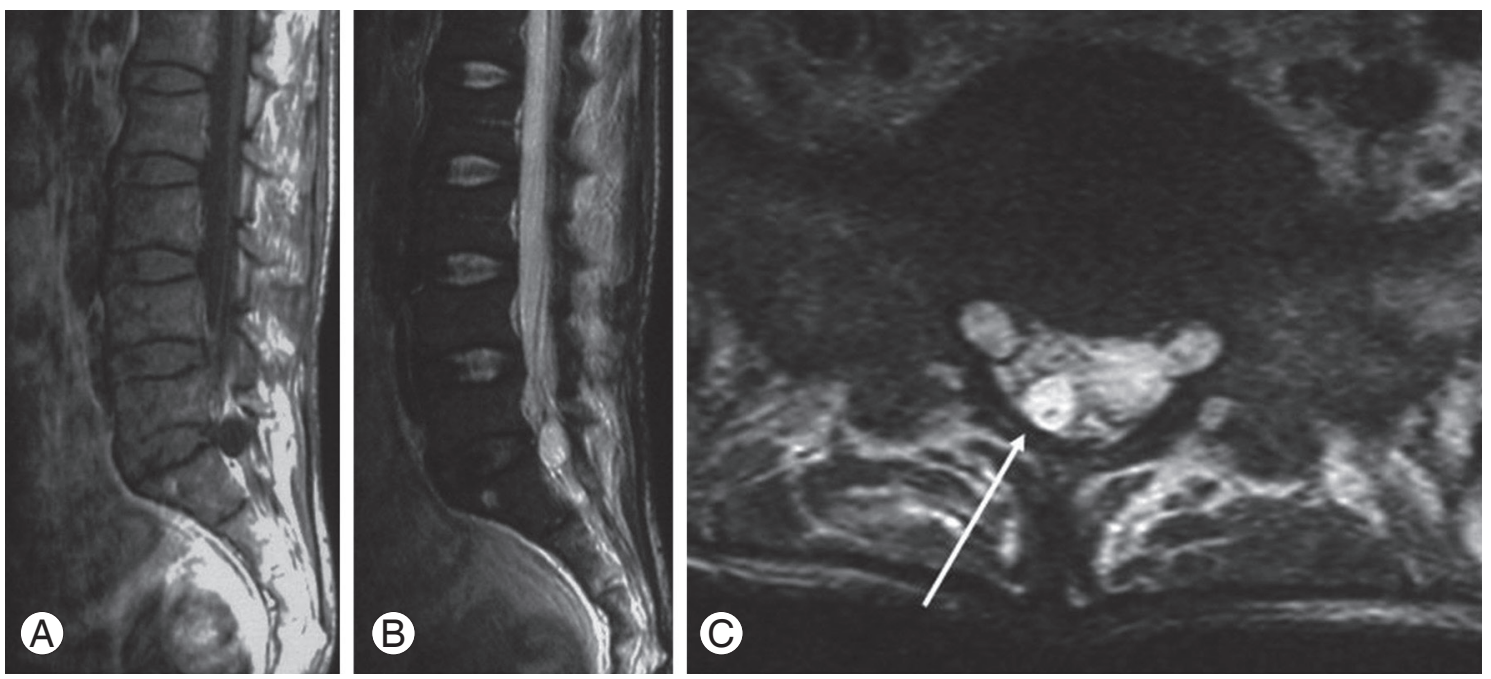

Fig. 1. A plain magnetic resonance imaging (MRI) revealed at least two perineural cysts or bilateral S2 root cysts at the $L 5 / S 1$ level. The white arrow indicates the right S2 root cyst. (A) MRI T1 sugittal. (B) MRI T2 sugittal. (C) MRI T2 axial.
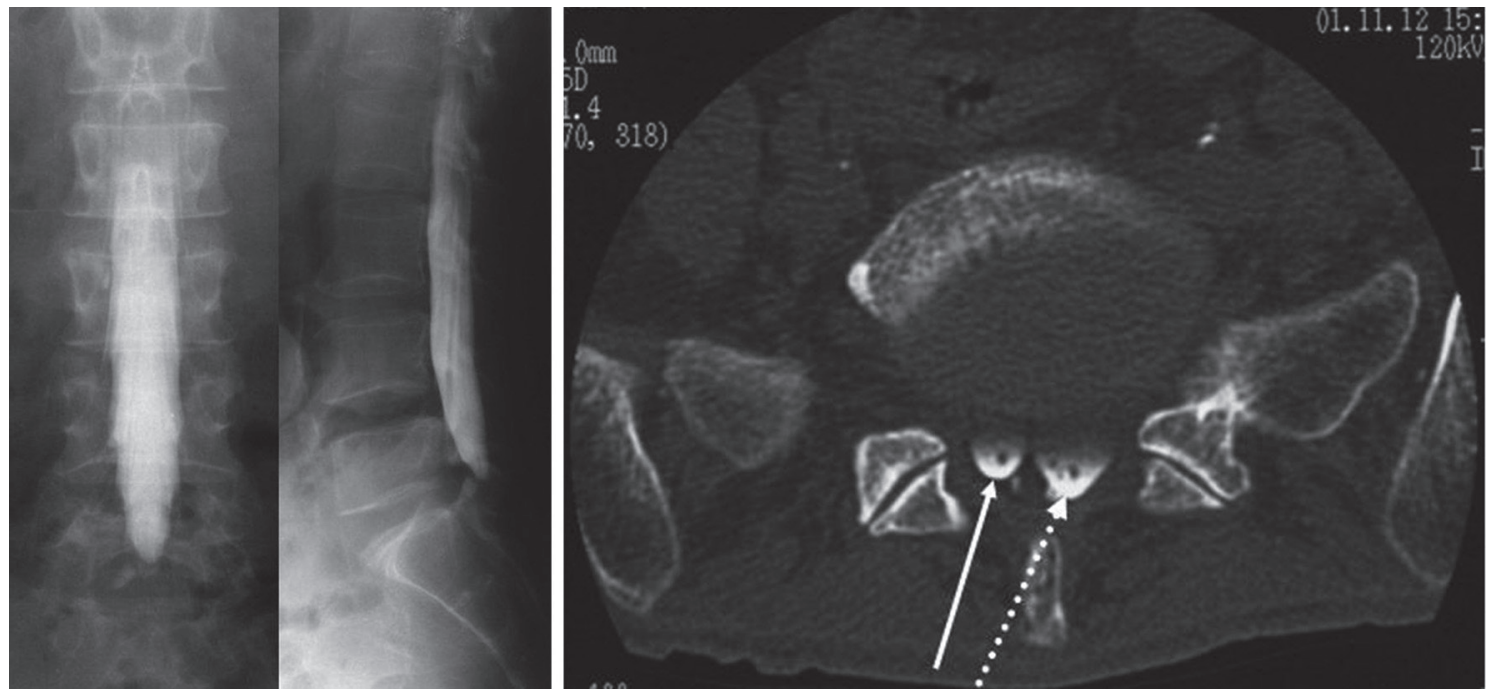

Fig. 2. Myelography showed a blockade of the contrast medium. Computed tomography taken after the myelography showed delayed filling of the right S2 root cysts and inflated dural sheath which contains two filaments. A white arrow indicates the right S2 root cyst. A dotted white arrow indicates left S2 or S3 root cysts as it contains two filaments. 
in order to decompress whole root cysts and sampled spinal fluid captured in left-side S2 root cyst then irrigated it with extracellular fluid. The intraoperative finding gave the impression that the blockage of the spinal flow due to stenosis had been alleviated (Fig. 3).

Sensation loss and urinary incontinence caused by sphincter disturbance subsided gradually and on the fifth postoperative day, her symptoms had almost disappeared. However, the residual existence of analgesics was not detected in the sampled spinal fluid.

\section{Discussion}

Symptomatic cysts are uncommon and the usual symptoms are pain or radiculopathy $[1,3]$. Some authors reported that it is linked with the urinary incontinence

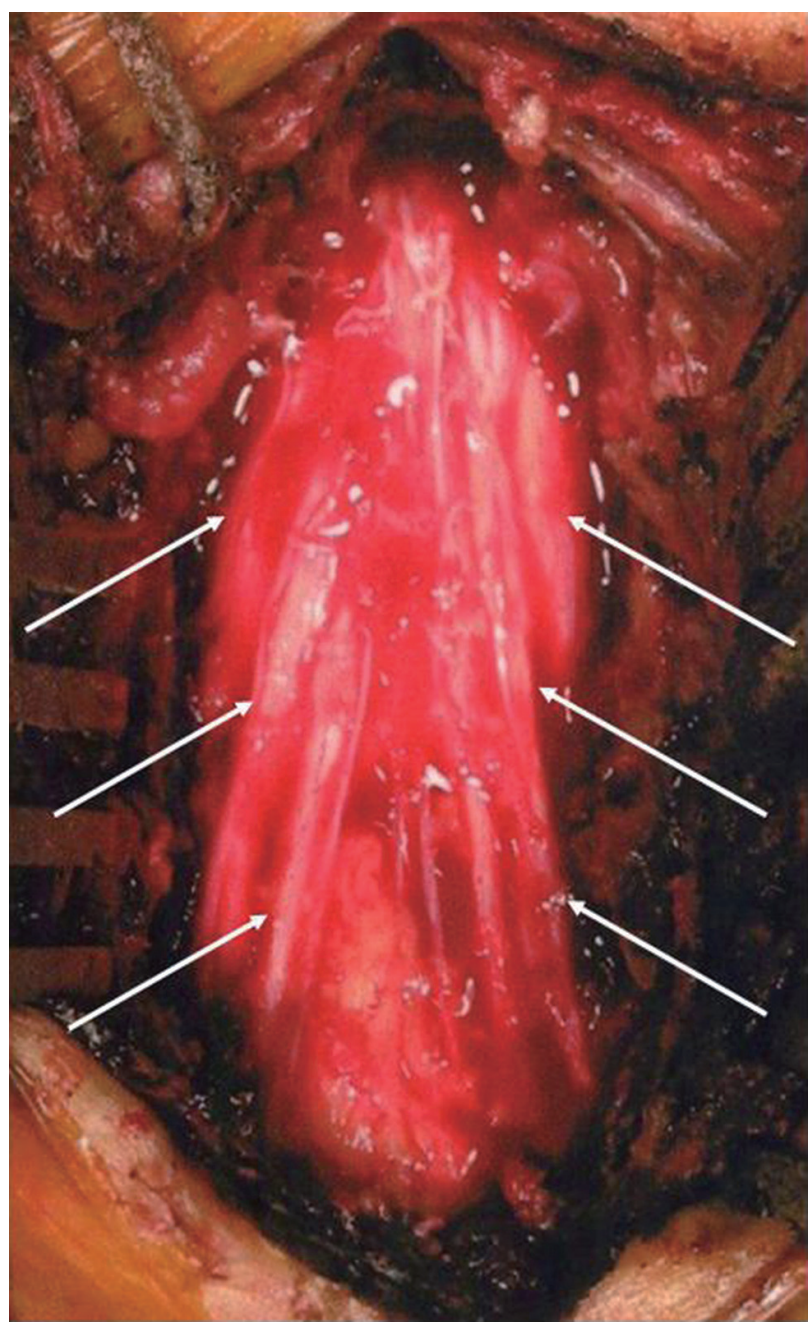

Fig. 3. Blockage of the spinal flow due to stenosis which seemed alleviated after decompression and irrigation. Symmetric white arrows indicate S2, S3, and S4 roots from above. occurrence $[4,5]$. In our hemodialysis case, cauda equine syndrome was not detected until she the patient reported a lack of sensation in the perianal area. Most authors have proposed microsurgical fenestration and imbrication for the treatment of the symptomatic perineural cysts $[3,6]$. However, a laminectomy with cyst irrigation was performed as we concluded that sphincter palsy was caused by the volume effect or analgesic effect as some authors claimed that etiology of the root cyst was explained by valve theory [6]. We believe that our surgical choice was right in 2001. However, advanced computed tomography (CT) can provide clinicians with accurate images. In 2004, Lee et al. [7] claimed that CT-guided percutaneous aspiration of a root cyst is an important diagnostic and prognostic procedure done prior to definitive operative treatment. Hence, clinicians should choose CT-guided percutaneous aspiration and irrigation as a first salvaging option before deciding on surgical intervention should clinicians encounter a similar case where spinal anesthesia or spinal-epidural anesthesia results in vesicorectal dysfunction.

It is well known that diabetes mellitus patients often have complications of aneurologic bladder which, in healthy patients, could possibly be the earliest indicator of vesicorectal dysfunction [8]. Her neurological status in terms of defecation function must have been susceptible to poor conditions, including hypotension or being bedridden. Hence, a volume effect or prolonged analgesic effect on the S2-4 root must have been quite catastrophic to cause paralysis. Since this was a case of an anuric maintenance hemodialysis patient, a diagnosis related to vesicorectal dysfunction was delayed. The patient and her medical staff could not detect the earliest indicator of vesicorectal dysfunction.

\section{Conflict of Interest}

No potential conflict of interest relevant to this article was reported.

\section{References}

1. Araki Y, Tsukaguchi I, Ishida T, et al. MRI of symptomatic sacral perineural cyst. Radiat Med 1992;10:250-2.

2. Ishii K, Yuzurihara M, Asamoto S, Doi H, Kubota M. A huge presacral Tarlov cyst: case report. J Neurosurg 
Spine 2007;7:259-63.

3. Mummaneni PV, Pitts LH, McCormack BM, Corroo JM, Weinstein PR. Microsurgical treatment of symptomatic sacral Tarlov cysts. Neurosurgery 2000;47:748.

4. Nicpon KW, Lasek W, Chyczewska A. Cauda equina syndrome caused by Tarlov's cysts: case report. Neurol Neurochir Pol 2002;36:181-9.

5. Ruibal Moldes M, Sanchez Rodriguez-Losada J, Lopez Garcia D, Casas Agudo V, Janeiro Pais JM, Gonzalez Martin M. Tarlov cyst and symptomatic bladder disfuction. Actas Urol Esp 2008;32:1035-6.

6. Tanaka M, Nakahara S, Ito Y, et al. Surgical results of sacral perineural (Tarlov) cysts. Acta Med Okayama 2006;60:65-70.

7. Lee JY, Impekoven P, Stenzel W, Lohr M, Ernestus RI, Klug N. CT-guided percutaneous aspiration of Tarlov cyst as a useful diagnostic procedure prior to operative intervention. Acta Neurochir (Wien) 2004;146:667-70.

8. Ross MA. Neuropathies associated with diabetes. Med Clin North Am 1993;77:111-24. 